\title{
A New Algorithm for the Distributed RWA Problem in WDM Networks Using Ant Colony Optimization
}

\author{
Víctor M. Aragón, Ignacio de Miguel, Ramón J. Durán, Noemí Merayo, \\ Juan Carlos Aguado, Patricia Fernández, Rubén M. Lorenzo, and Evaristo J. Abril \\ Dpt. Signal Theory, Communications and Telematic Engineering, University of Valladolid, \\ Campus Miguel Delibes, 47011 Valladolid, Spain \\ Tel: +34 983423000 ext. 5574, Fax: +34 983423667 \\ varafer@coit.es, ignacio.miguel@tel.uva.es
}

\begin{abstract}
We present a new algorithm based on ant colony optimization to solve the dynamic routing and wavelength assignment problem in a distributed manner. The algorithm uses the ant colony optimization metaheuristic to obtain updated information about the network state, which is then used to find the routes and wavelengths to establish new connections through an adaptive process able to deal with dynamic changes in network state. By means of simulation we show that the algorithm leads to lower blocking probability and lower mean setup time than other distributed algorithms.
\end{abstract}

Keywords: Optical networks, Wavelength Division Multiplexing (WDM), Dynamic Routing and Wavelength Assignment (RWA), distributed systems, Ant Colony Optimization (ACO).

\section{Introduction}

Ants are social insects that despite not having a remarkable individual intelligence are able to coordinate themselves to make complex tasks using chemical signals that modify the behavior of the individuals. The most interesting feature, from the point of view of telecommunications, is the method that they follow to find the shortest path between two points. Each individual deposits a chemical sign, called pheromone, as it moves; and each ant has a certain tendency to follow those trails presenting higher intensity of such a substance. Hence, due to the interaction of many individuals, the pheromone tends to concentrate in the shortest paths. This method can be adapted for the resolution of multiple problems represented through graphs, leading to a metaheuristic known as Ant Colony Optimization, ACO [1].

ACO has been used to solve the problem of routing in telecommunication networks based on circuit switching and on packet switching. In the last years, ACO has also been used to solve the routing and wavelength assignment (RWA) problem in optical networks. In particular, for the dynamic problem, where all-optical connections (lightpaths) are established and released on user demand, there are several proposals [2-4], all of them using centralized control. In these works, the artificial ants that travel across the network are only used to configure the routing tables in the nodes, helping to solve only a part of the RWA problem. 
In this paper, we present a novel algorithm to solve the dynamic RWA problem in an optical network with distributed control, where each network node cooperates with the others to solve the problem. The new algorithm, which will be described in Section 2, uses ACO not only to configure the routing tables in the nodes, but also to collect information about wavelength availability in the links of the network, thereby providing complete information about the RWA problem, and leading to lower blocking probability and lower mean setup time than other distributed algorithms, as we will show in Section 3 of the paper.

\section{The New Algorithm, DACOS}

The new algorithm, named DACOS (Distributed Ant Colony Optimization Scheme for the RWA problem), is designed to operate in an optical network without wavelength converters where bidirectional connections are dynamically established and released. The algorithm comprises two parts: the ACO system, which is in charge of building local routing tables with information about wavelength availability; and the reservation system, which processes establishment and release requests. DACOS involves the transmission of control packets through the network, which could be transported using a specific wavelength reserved for that aim.

\subsection{The ACO System: Building the Local Routing Tables}

The first part of DACOS is an ACO system which consists in the network nodes periodically sending artificial ants (management packets) to other nodes. These artificial ants imitate the behavior of natural ants, and so choose the following node of their route according to the concentration of artificial pheromone, which is a value associated to each link for each destination node (Fig. 1). When an ant arrives at a node, the pheromone is increased in the link by which it arrived, but only for the nodes that it has previously visited (as a way of indicating that those nodes previously traversed by the ant can be reached going through that link). The pheromone is increased as a function of both the distance (in number of hops) traversed by the ant from each of those nodes, and the availability of common wavelengths in the links traversed by the ant. For instance, let us consider the network shown in Fig. 1, and assume that an ant departs from node 4 , traverses node 5 and arrives at node 1 . Since the ant arrives through link $l_{5}$, and has visited nodes 4 and 5 in its trip, the pheromone would be increased for that link and those destinations (represented in bold in the table in Fig. 1). The pheromone amounts in the table are normalized for each row, so their sum must be one for each destination node. In this way, the pheromone amounts are used as a probability distribution to choose the outgoing link that the artificial ants arriving at that node will traverse, and - as we will explain later-, as a way to estimate the most appropriate next node for a specific connection request. As an example of the first issue, if an ant is processed at node 1 and has node 2 as destination, the ant will be very probably forwarded through link $l_{1}$, as it has the largest amount of pheromone for the row corresponding to the destination node 2 . However, with the aim of expanding the exploration of new routes across the 
network, a small percentage of artificial ants select the next node to visit according to a uniform distribution, instead of having more probability to follow the link with the largest amount of pheromone.

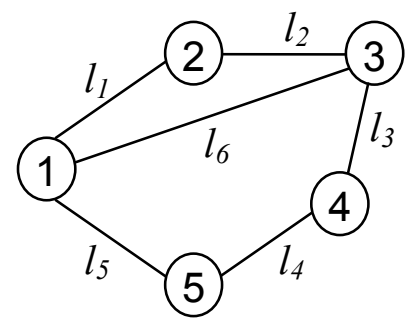

Pheromone table at node 1

\begin{tabular}{|c|c|c|c|}
\hline \multirow{2}{*}{ Destination } & \multicolumn{3}{|c|}{ Link } \\
\cline { 2 - 4 } & $l_{1}$ & $l_{5}$ & $l_{6}$ \\
\hline Node 2 & 0.6 & 0.1 & 0.3 \\
\hline Node 3 & 0.2 & 0.1 & 0.7 \\
\hline Node 4 & 0.1 & $\mathbf{0 . 5}$ & 0.4 \\
\hline Node 5 & 0.1 & $\mathbf{0 . 6}$ & 0.3 \\
\hline
\end{tabular}

Fig. 1. Sample network and example of pheromone table at node 1.

The artificial ants are also used to configure the routing tables of the nodes by combining the pheromone tables with information about wavelength availability. In this way, the routing tables indicate the most appropriate outgoing link for establishing a connection with each possible destination (that having more pheromone), and contain updated data on the availability of each wavelength to reach each destination. To accomplish this latter issue, an ant not only collects information while traversing the network such as the availability of common wavelengths in that route, but it also retrieves information about wavelength availability from the nodes of its route that was deposited by previous ants following alternate routes, and propagates this information to the next nodes that it visits.

\subsection{The Reservation System: Processing Establishment and Release Requests}

The second part of DACOS is a reservation system, which is in charge of processing establishment and release requests. When a node desires to establish a lightpath with another node, the information of the local routing table is used to select both the optimal outgoing link and the wavelength that has more probability to be available until the destination node. First of all, the link with the highest pheromone value (for the destination node) is selected. Then, the routing table is again consulted to determine the set of wavelengths that are available until the destination node of the connection through that link (or more precisely, that were available according to the information carried by the last ant that arrived at this node from the destination node). If there are several wavelengths available, the one selected is that which is free in a lower number of links connected to the source node, as a heuristic to minimize the blocking probability of future connections traversing that node. If at this point there are several wavelengths with the same number, one of them is chosen randomly. If there are no available wavelengths, that link is ruled out and the same process is repeated on the next link with highest pheromone value. Once the outgoing link and the wavelength for the connection have been selected in the source node, a control 
packet is sent to the following node, and the selected wavelength is reserved. Since the network does not have wavelength converters, the next node must select an outgoing link where the selected wavelength is available. First of all, the link with the highest pheromone value for the destination node is selected. Then, using the information of the local routing table, it is checked whether the selected wavelength for the connection is expected to be available in each link across some route to the destination node. If found, that link is chosen to send the control packet. Otherwise, that link is ruled out and the next link with highest pheromone value is checked. The process that we have just mentioned is repeated in the following nodes of the route until the destination node is reached.

It is worthy to note that the wavelength is chosen by the source node and it is reserved in all the links that the control packet crosses. However, the sequence of nodes that will be traversed by the control packet (and hence used to establish the connection) is not predetermined in the source node but selected according to the availability of the wavelength chosen for the connection. Moreover, each control packet of the reservation system includes an ant, so that it also updates the routing tables of the nodes when moving across the network.

If the connection cannot be established due to lack of resources, a probe packet is sent from the point where the connection gets blocked towards the destination node, and a release packet is sent backwards to free the reserved wavelengths in the visited links. When the probe packet reaches the destination, the connection establishment process can be repeated from there (since connections are bidirectional, they can be established in any direction). In this way, if a connection is blocked due to wavelength unavailability, it can be reattempted for a number of times if required, at the expense of increasing the setup time of the connection.

On the other hand, when a connection is to be released, a control packet is sent along the route in order to free all the reserved resources.

\section{Performance Evaluation}

In order to evaluate the performance of DACOS in terms of both blocking probability and setup time, we have compared it by means of simulation with two distributed RWA algorithms. The first one uses Fixed Routing (FR), where the routes are precalculated using an ACO method that searches the shortest routes. For wavelength assignment a backward reservation method [5] is used together with the First Fit (FF) heuristic [6]. From now on, this algorithm will be named FR-FF. The second algorithm is a modified version of the ACO-based algorithm presented by Ngo et al. [3]. The original algorithm used centralized control, so we have adapted it to a distributed environment. Like the previous algorithm, a backward reservation method has been implemented for wavelength assignment together with the First Fit (FF) scheme. Henceforth we will name it Ngo algorithm. In DACOS, a maximum of four attempts were allowed for each connection request.

The simulator has been implemented in OPNET Modeler [7], and the algorithms have been evaluated in the NSFNet topology [8] in terms of blocking probability, and mean and maximum setup time. Connection requests were generated at each node 
following a Poisson process with arrival rate $\lambda_{\text {node }}$, and the destination nodes for the connections were randomly chosen according to a uniform distribution. Thus, the total arrival rate in the whole network was $\lambda_{\text {total }}=N \cdot \lambda_{\text {node }}$, where $N$ is the number of network nodes. The connection holding time was exponentially distributed with mean $T$. Therefore, the normalized traffic load is given by $\lambda_{\text {total }} T /[N \cdot(N-1)]$.

\subsection{Blocking Probability}

Fig. 2 shows the mean blocking probability for DACOS, FR-FF and Ngo algorithms, for different numbers of wavelengths per link. It can be seen that DACOS outperforms the other algorithms, and as the network links become saturated, its blocking probability converge with the other ones. For a moderate load and an adequate number of wavelengths, between 16 and 32, the blocking probability of DACOS is around $10^{-3}$, while the other algorithms have a blocking probability higher than $10^{-2}$. Fig. 2 also shows that there is an interval of traffic loads for which the blocking probability of the FR-FF and Ngo algorithms decrease. Due to the method that we have used to increase the traffic load in the simulation, which has consisted in extending the duration of the connections, the network state changes less frequently, and FR-FF and Ngo algorithms improve their performance in that scenario. That is due to the backward reservation method, which leads to lower backward blocking probability since the information about wavelength availability carried by the control packet requesting the connection, and received by the final node, is less outdated in these scenarios [5]. Obviously, if the traffic load is further increased, the lack of resources becomes dominant and the blocking probability rises again.

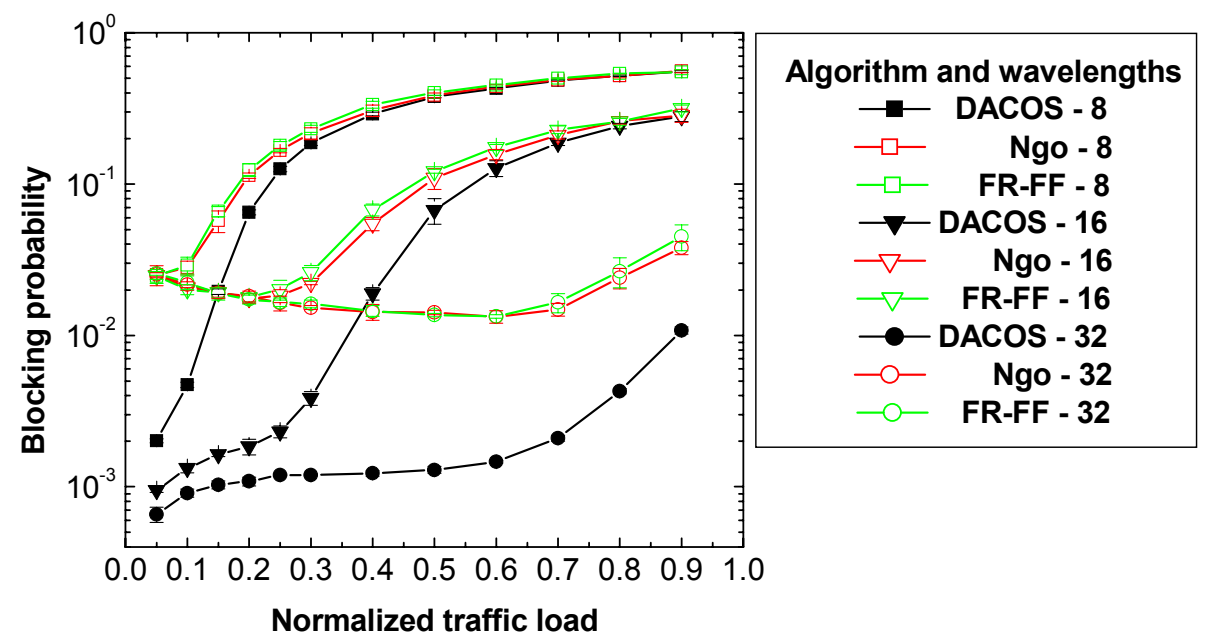

Fig. 2. Blocking probability for DACOS and other distributed algorithms. $95 \%$ confidence intervals are shown. 


\subsection{Mean Setup Time}

Fig. 3 shows the mean setup time, i.e., the mean time elapsed between the generation of a connection request at a node and the moment in which both end nodes receive confirmation of the connection being completely established. DACOS again outperforms the other algorithms. The mean setup time for DACOS is around $28 \mathrm{ms,}$ while the others are around $40 \mathrm{~ms}$ in the low traffic load region. DACOS gets a lower mean setup time because in FR-FF and Ngo three trips of control packets are always required to establish a connection (one to check wavelength availability, one to reserve a wavelength, and another one to confirm the reservation) whereas in DACOS only two trips are required if the connection is established in the first attempt (one to reserve a wavelength and another one to confirm the reservation). Fig. 3 also shows that as the traffic load increases, the mean setup time decreases. The reason is that as links become saturated, the longest connections are the most likely ones to be blocked, so the mean time to establish a connection decreases as the connections become shorter (and hence the control packets travel shorter distances). However, the mean setup time initially increases for DACOS, because when the network becomes slightly saturated, it selects longer routes that are less congested, in an attempt to not to increase the blocking probability. Due to this behavior a peak in the figure can be noticed, showing a displacement to higher traffic loads as the number of wavelengths increases.

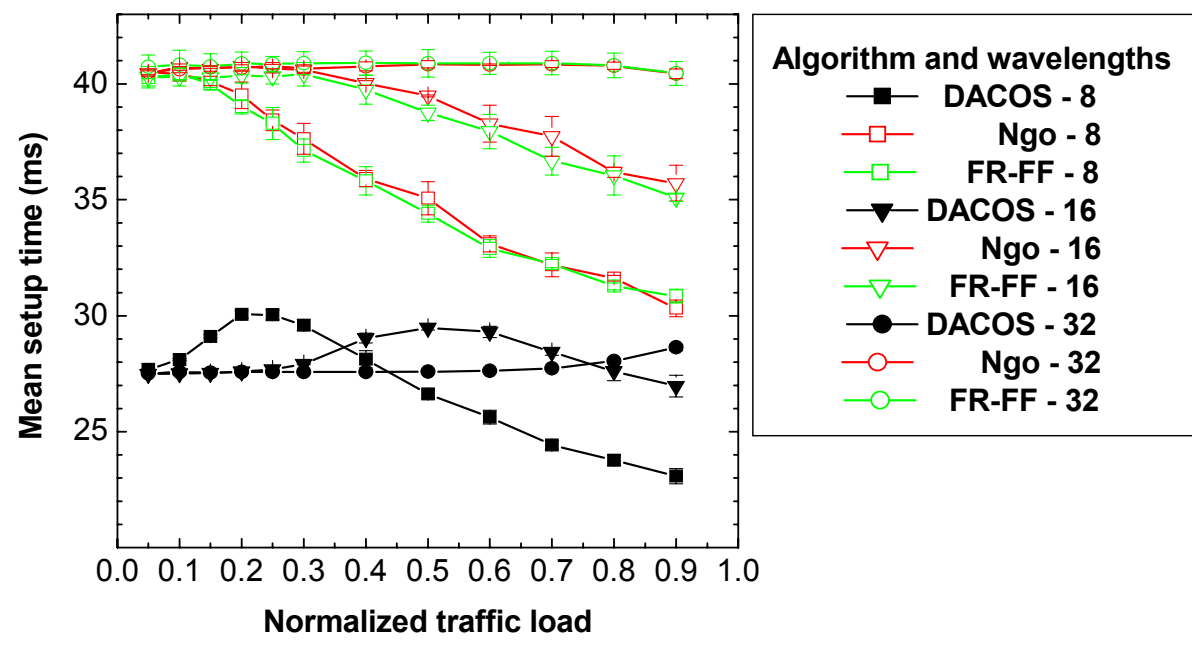

Fig. 3. Mean setup time for DACOS and other distributed algorithms. 95\% confidence intervals are shown. 


\subsection{Maximum Setup Time}

Fig. 4 shows the maximum setup time, i.e., the time elapsed between the generation of a connection request at a node and the moment in which both end nodes receive confirmation of the connection being completely established in the worst case. DACOS presents a higher and more unpredictable maximum than the other two algorithms. FR-FF and Ngo get a very stable worst case around 90-100 ms, because they always require three trips of control packets to establish a connection. Thus, the only affecting factor is the length of the path. For this reason, FR-FF yields a slightly lower maximum, since it always searches for the shortest paths, while Ngo lets longer less congested paths to be used to establish the connections. DACOS, besides being more flexible with the length of the paths, allows up to four attempts for each connection request, so the setup time can vary notably and be high, around $180 \mathrm{~ms}$ with $95 \%$ confidence intervals even wider than $60 \mathrm{~ms}$. Like in the mean setup time case, a peak can be noticed in DACOS graph, showing a displacement to higher traffic loads as the number of wavelengths increases. On the other hand, it is worthy to note that, if required, the maximum number of connection attempts in DACOS can be reduced in order to decrease the maximum setup time and its variability.

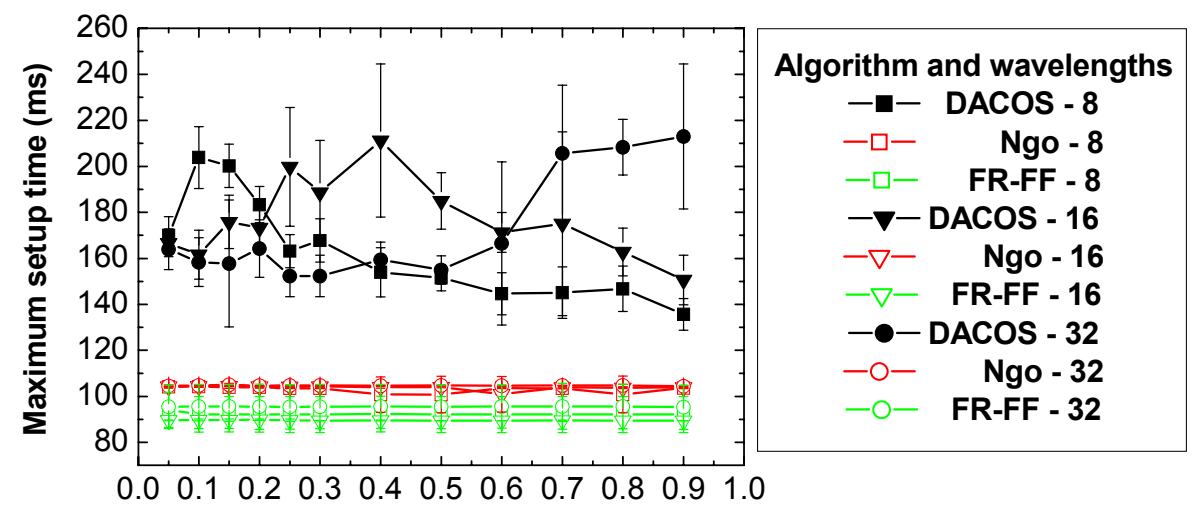

Normalized traffic load

Fig. 4. Maximum setup time for DACOS and other distributed algorithms. $95 \%$ confidence intervals are shown.

\subsection{Impact of the Number of Connection Attempts}

The FR-FF and Ngo algorithms can only attempt to establish the connection once. However, DACOS can repeat the process several times if required (in the analyzed scenario, up to four times). Thus, Fig. 5 shows the mean number of connection attempts used by DACOS to establish a connection. As shown in the figure, the higher the number of wavelengths in the links, the lower the mean number of connection attempts that DACOS requires. Even when considering the worst case, with eight wavelengths, the mean is low (1.14), so that most of the connections are established in the first attempt. This fact proves that the algorithm is very efficient in 
using the information gathered by the artificial ants to select the wavelength and the route of the connections.

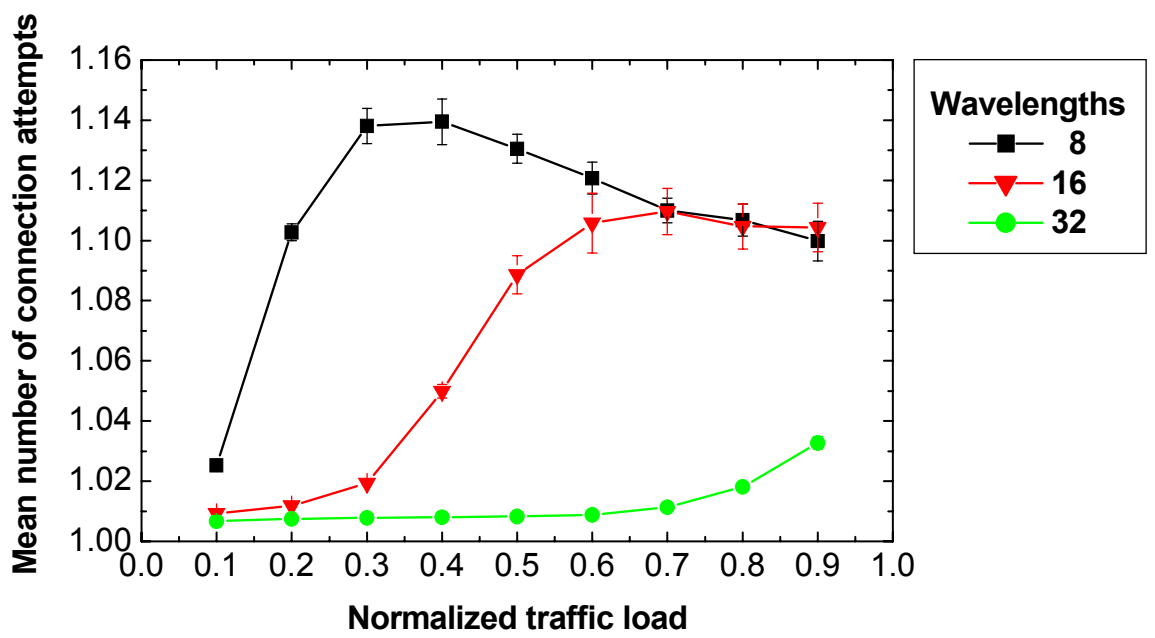

Fig. 5. Mean number of connection attempts for DACOS. 95\% confidence intervals are shown.

As a further view about this issue, Fig. 6 shows the blocking probability for DACOS depending on the maximum number of connection attempts, when equipping the NSFNet with 16 wavelengths. Fig. 7 shows the mean setup time for that scenario.

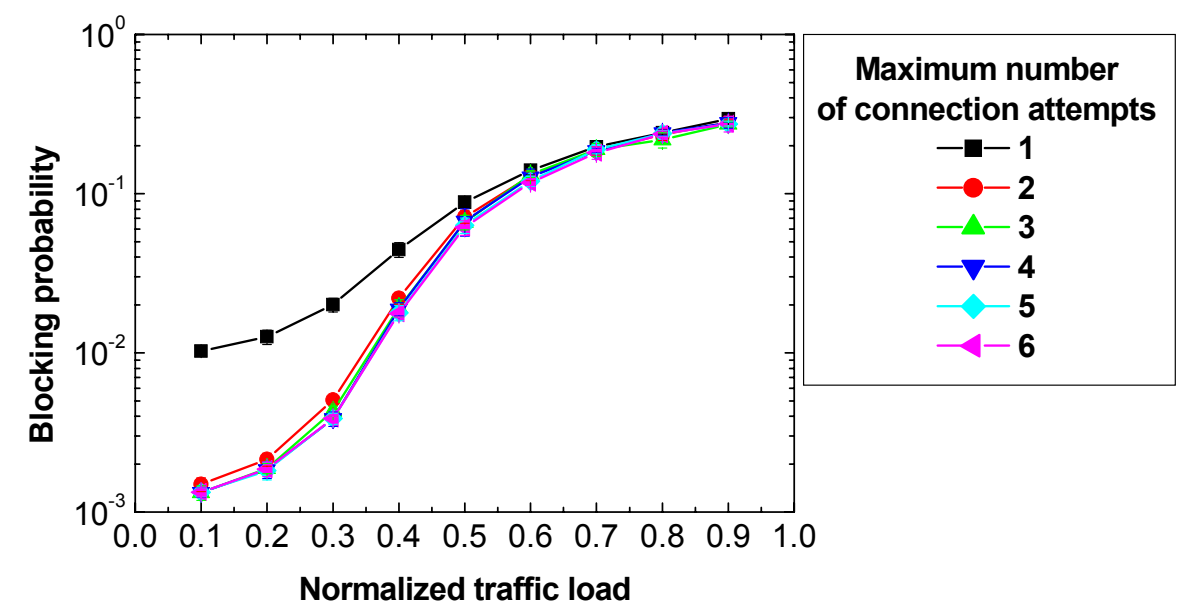

Fig. 6. Blocking probability for DACOS depending on the maximum number of connection attempts, with 16 wavelengths. 95\% confidence intervals are shown.

As shown in Fig. 6, the blocking probability obtained with DACOS, when only one attempt is allowed to establish a connection, is similar to that obtained with FR-FF 
and Ngo algorithms (Fig. 2). However, when setting a maximum of two attempts, there is a significant decrease in blocking probability, getting very close to the results obtained with a maximum of four attempts (which was the value used in the simulation results previously shown). In fact, as shown in the figure, there is no significant improvement for further increases on the maximum number of attempts.

On the other hand, allowing several attempts has very little penalty on the mean setup time, as shown in Fig. 7. In fact, even with six attempts (Fig. 7), the mean setup time is lower than that obtained with FR-FF and Ngo algorithms (Fig. 3).

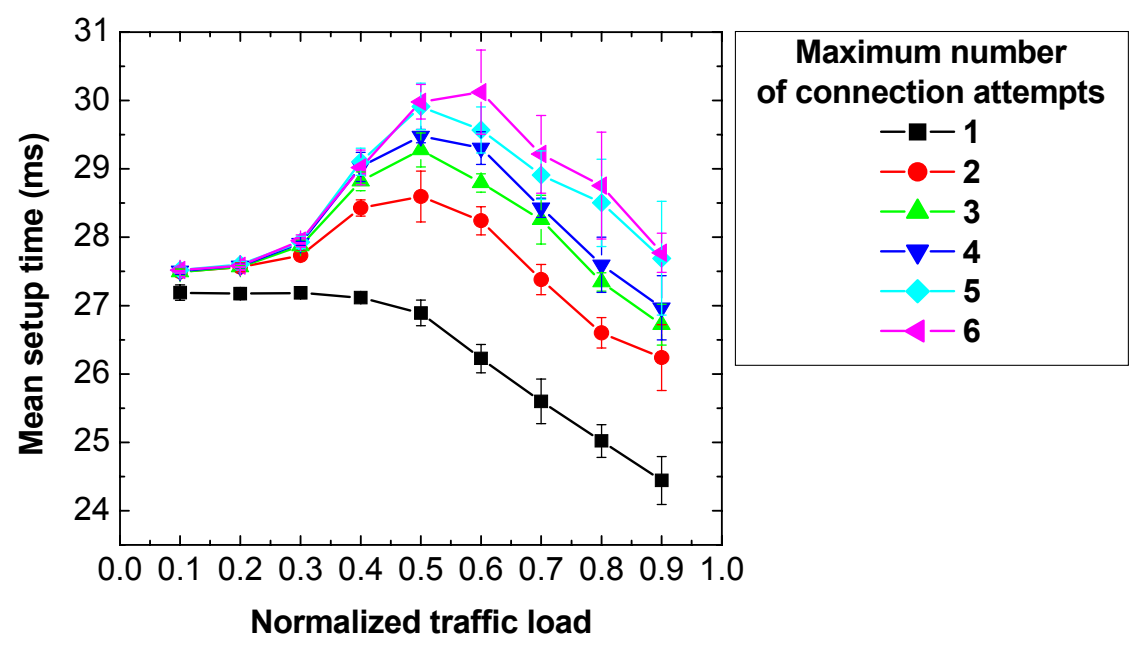

Fig. 7. Mean setup time for DACOS depending on the maximum number of connection attempts, with 16 wavelengths. 95\% confidence intervals are shown.

In summary, if DACOS is configured with a single attempt, the performance in terms of blocking is similar to that of Ngo and FR-RR, but much faster, as FR-FF and Ngo require three trips of control packets to establish a connection, but DACOS only requires two. If DACOS is configured with more than one attempt, the blocking probability significantly decreases while still being notably faster than Ngo and FR-RR.

\section{Conclusions}

We have proposed a new algorithm, DACOS, which efficiently solves the dynamic and distributed RWA problem by means of ant colony optimization.

DACOS presents some completely original characteristics when compared with other methods based on ant colony optimization. The ants are not only limited to make the tasks of configuring routing tables in the network, but they also provide updated information about wavelength availability. In this way, the reservation system takes advantage of that information in the source node of each connection to 
select both the next node and the wavelength with highest probability of being available in all the links until the destination node. While the wavelength is selected by the source node, the route is not predetermined, but it is adaptively selected by the intermediate nodes, using more updated information than that available in the source node, and thus making possible to cope with changes in the state of the network, by taking alternative routes to establish the connection when required. Moreover, a hybrid reservation mechanism combining ideas from forward and backward reservation is used, which allows to make manifold attempts of connection if needed.

We have shown that DACOS outperforms other distributed and dynamic RWA algorithms in terms of both blocking probability (over an order of magnitude in the NSFNet) and mean setup time (about 30\% faster in that topology). However, DACOS also presents a higher and more unpredictable maximum setup time, mainly due to its variable number of connection attempts. That technique leads to a significant decrease of the blocking probability with very little increase in the mean setup time. That is due to the fact that most connections are established in the first attempt (thereby having very little setup time as only two trips of control packets are involved, while other less efficient algorithms require three), and additional connections, that otherwise would be blocked, are established (of course at the expense of higher setup time). For those reasons, DACOS gets lower blocking probability while still being faster than other algorithms.

Acknowledgments. This work has been supported by the Spanish Ministry of Education and Science and Technology (Ministerio de Educación y Ciencia) under grant TEC2005-04923 and by Consejería de Educación de la Junta de Castilla y León under grant VA028B06.

\section{References}

1. Bonabeau, E., Dorigo, M., Theraulaz, G.: Inspiration for Optimization from Social Insect Behaviour. Nature, Vol. 406 (2000) 39-42

2. Garlick, R.M., Barr, R.S.: Dynamic Wavelength Routing in WDM Networks via Ant Colony Optimization. In: Proceedings ANTS'02 (2002) 250-255

3. Ngo, S.H., Jiang, X.H. and Horiguchi, S.: An Ant-Based Approach for Dynamic RWA in Optical WDM Networks. Photonic Network Communications, Vol. 11 (2006) 39-48

4. Sousa, G., Waldman, H.: Evaluation of an Ant-based Architecture for All-Optical Networks. In: Proceedings ONDM'06. Copenhagen, Denmark, (2006)

5. Lu, K., Xiao, G. and Chlamtac, I.: Analysis of Blocking Probability for Distributed Lightpath Establishment in WDM Optical Networks. IEEE/ACM Transactions on Networking, Vol. 13, No. 1 (2005) 187-197

6. Zang, H., Jue, J.P., Mukherjee, B.: A Review of Routing and Wavelength Assignment Approaches for Wavelength-Routed Optical WDM Networks. Optical Networks Magazine, Vol. 1, No. 1 (2000) 47-60

7. OPNET Modeler, http://www.opnet.com

8. Baroni, S., Bayvel, P.: Wavelength Requirements in Arbitrarily Connected WavelengthRouted Optical Networks. Journal of Lightwave Technology, Vol. 15, No. 2 (1997) 242-251 